\title{
Campylobacter polysaccharide capsules: virulence and vaccines
}

Patricia Guerry ${ }^{1}$, Frédéric Poly ${ }^{1}$, Mark Riddle ${ }^{1}$, Alexander C. Maue ${ }^{1}$, Yu-Han Chen ${ }^{2}$ and Mario A. Monteiro ${ }^{2}$

${ }^{1}$ Enteric Diseases Department, Naval Medical Research Center, Silver Spring, MD, USA

2 Department of Chemistry, University of Guelph, Guelph, ON, Canada

\section{Edited by:}

Alain Stintzi, Ottawa Institute of Systems Biology, Canada

\section{Reviewed by:}

Mark Estes, University of Georgia, USA

Christine M. Szymanski, University of Alberta, Canada

\section{*Correspondence:}

Patricia Guerry, Enteric Diseases

Department, Naval Medical Research Center, 503 Robert Grant Avenue, Silver Spring, MD 20910, USA. e-mail: patricia.guerry@med.navy.mil

Campylobacter jejuni remains a major cause of bacterial diarrhea worldwide and is associated with numerous sequelae, including Guillain Barré Syndrome, inflammatory bowel disease, reactive arthritis, and irritable bowel syndrome. C. jejuni is unusual for an intestinal pathogen in its ability to coat its surface with a polysaccharide capsule (CPS). These capsular polysaccharides vary in sugar composition and linkage, especially those involving heptoses of unusual configuration and $O$-methyl phosphoramidate linkages. This structural diversity is consistent with CPS being the major serodeterminant of the Penner scheme, of which there are $47 C$. jejuni serotypes. Both CPS expression and expression of modifications are subject to phase variation by slip strand mismatch repair. Although capsules are virulence factors for other pathogens, the role of CPS in $C$. jejuni disease has not been well defined beyond descriptive studies demonstrating a role in serum resistance and for diarrhea in a ferret model of disease. However, perhaps the most compelling evidence for a role in pathogenesis are data that CPS conjugate vaccines protect against diarrheal disease in non-human primates. A CPS conjugate vaccine approach against this pathogen is intriguing, but several questions need to be addressed, including the valency of CPS types required for an effective vaccine. There have been numerous studies of prevalence of CPS serotypes in the developed world, but few studies from developing countries where the disease incidence is higher. The complexity and cost of Penner serotyping has limited its usefulness, and a recently developed multiplex PCR method for determination of capsule type offers the potential of a more rapid and affordable method. Comparative studies have shown a strong correlation of the two methods and studies are beginning to ascertain CPS-type distribution worldwide, as well as examination of correlation of severity of illness with specific CPS types.

\section{Keywords: Campylobacter, capsules, capsule conjugate vaccines, virulence}

\section{INTRODUCTION}

Campylobacter jejuni, one of the most common causes of bacterial diarrhea worldwide, is biologically distinct from other enteric pathogens, such as Salmonella, Shigella, and Vibrio. A member of the epsilon proteobacteria, C. jejuni is more similar to Gram negative mucosal pathogens such as Haemophilus influenzae and Neisseria meningitidis in that it is microaerophilic, naturally transformable, and encapsulated. The polysaccharide capsule (CPS), which is the topic of this review, is unique for an enteric pathogen, and the C. jejuni capsular polysaccharides are unique compared to most others.

\section{CAMPYLOBACTER CAPSULES: STRUCTURES AND GENETICS}

During the 1990s, Aspinall and co-workers discovered that Campylobacter species (C. jejuni, C. coli, and C. lari) exposed polysaccharides (PSs) that were considered to be O-chain PS regions of cell-wall lipopolysaccharides (LPSs; Aspinall et al., 1992, 1993, 1995a,b; McDonald, 1993; Aspinall, 1998). However, structural data obtained from some Campylobacter species showed that these moieties were not associated with a LPS component, but were of the same type as teichoic acid PSs, as in C. jejuni serotype
HS1 (for Heat Stable serotype; McDonald, 1993) and capsule PSs (CPSs), as in C. lari (Aspinall, 1998). Subsequently, genomic analysis provided confirmation that the observed PSs of $C$. jejuni were capsule (CPSs; Parkhill et al., 2000). Corcoran et al. (2006) demonstrated the phospholipid anchor in three CPS types (HS3, HS6, and HS23/36) was dipalmitoyl-glycerophosphate, with esterlinked hexadecanoic acids. Campylobacter species, like other Gram negative mucosal pathogens and unlike other enteric pathogens, express a CPS and lipooligosaccharide (LOS; core $\rightarrow$ lipid A) in lieu of a full length LPS (O-chain $\rightarrow$ core $\rightarrow$ lipid A). The Penner serotyping scheme is a passive slide hemagglutination that is based primarily on CPSs, although other structures, including LOS can contribute to serotype specificity (Penner and Hennessy, 1980; Preston and Penner, 1989; Karlyshev et al., 2000). A total of 23 serotypes were initially described in the original publication (Penner and Hennessy, 1980), and this was quickly extended to 47 serotypes for $C$. jejuni, although many are found in related, cross-reacting complexes.

The structure of eight CPS types of $C$. jejuni have been published (Aspinall et al., 1992, 1995c; Hannify et al., 1999; Muldoon et al., 2002; Karlyshev et al., 2005; McNally et al., 2005, 2007; Gilbert 
et al., 2007; Chen et al., 2008), and these vary in sugar composition and linkage. The expression of (i) heptoses of unusual configuration (i.e., altro, ido, gulo, talo) and (ii) $O$-methyl phosphoramidate $(\mathrm{MeOPN})$ are key structural markers of the capsules of Campylobacter species, especially C. jejuni (Figure 1). The structural complexity of the heptoses is further enhanced by the introduction of a deoxy function at the C- 6 position, in that, within a single CPS polysaccharide chain, it is common to observe the presence of the heptose and its complementary 6-deoxy-heptose, for example, D-glycero-D-altro-heptose and 6-deoxy-altro-heptose in C. jejuni strains that belong to serotype complex HS23/36 (Aspinall et al., 1993; Kanipes et al., 2006). MeOPN has been identified on most C. jejuni CPSs, although it is found attached in different linkages to different sugars in each (Karlyshev et al., 2005; McNally et al., 2007). MeOPN is also found in non-stoichiometric amounts, likely because of phase variation (see below).

Campylobacter jejuni capsules are assembled via an ABC transporter mechanism, similar to class 2 and class 3 capsules of $E$.

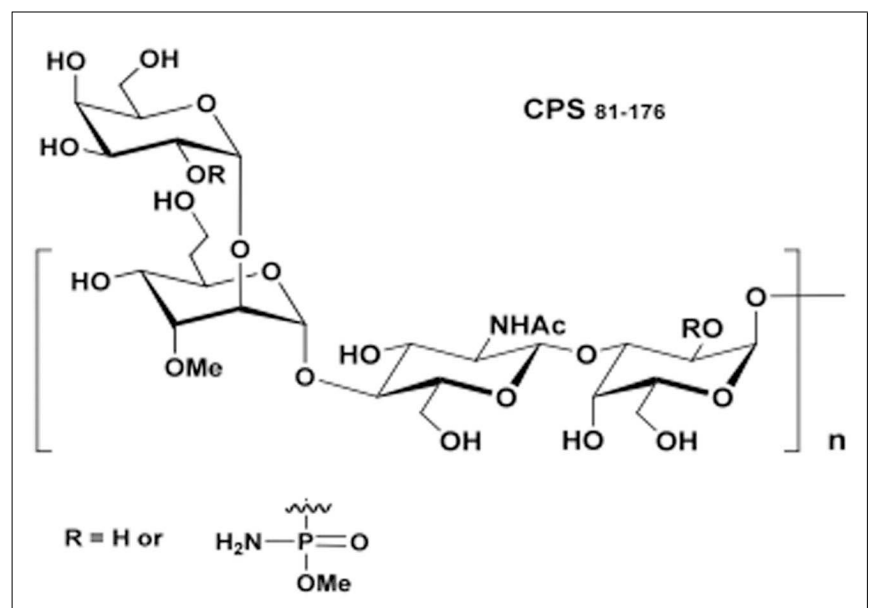

FIGURE 1 | Covalent structure of the CPS from $C$. jejuni strain 81-176 (serotype HS23/36). The trisaccharide repeating block is composed of $\rightarrow$ 3)-D-Galp-(1 $\rightarrow$ 2)-3-Me-6d- $\alpha$-D-altro-Hepp-(1 $\rightarrow$ 3)- $\beta$-D-GlcpNAc- $(1 \rightarrow$. The methylated heptose is sometimes substituted by

6-deoxy-altro-heptose. The O-methyl phosphoramidate side chain is connected to the $\mathrm{C}-2$ position of $\mathrm{Gal}$ in non-stoichiometric amounts (Kanipes et al., 2006). coli $\mathrm{K} 1$ and $\mathrm{K} 5, N$. meningitidis and $H$. influenzae, and the genetic organization of capsule genes in C. jejuni is similar to those found in these bacteria, as shown in Figure 2. Capsule genes in these groups are organized in three regions, where the conserved regions 1 and 3 are involved in capsule assembly and transport, and the variable region 2 encodes genes responsible for synthesis of the polysaccharides. The major difference between CPS groups 2 and 3 is the organization of the kps genes, and the presence of an additional gene involved in thermoregulation of capsule synthesis, $k p s U$, in group 2 capsule strains. C. jejuni is more similar to the group 3 capsules by its absence of the kps $U$ gene and apparent lack of thermoregulation (Stintzi, 2003), but appears to be a hybrid between groups 2 and 3 due to differences in gene organization (Figure 2). Although the kpsM gene of $81-176$ has been shown to functionally complement the corresponding mutation in E. coli K1 (Bacon et al., 2001), no functional studies of C. jejuni $k p s$ gene products have been reported. The function of the products of Class 2 and Class $3 \mathrm{kps}$ genes have been reviewed recently (Vimr and Steenbergen, 2009).

Region 2, located between $k p s C$ and $k p s F$ in $C$. jejuni, contains genes that are responsible for biosynthesis of specific polysaccharides (see Figure 3). Variability of this region reflects the array of CPS structures/Penner serotypes of C. jejuni. A total of 18 CPS loci from distinct Penner types have been sequenced to date (Parkhill et al., 2000; Pearson et al., 2004; Fouts et al., 2005; Karlyshev et al., 2005; Poly et al., 2011). Region 2 in these strains contains from 11 to 30 genes and ranges in size to from 11 to $34 \mathrm{~kb}$. Variation in this region reflects horizontal gene transfer, gene duplication, deletion, and fusion, all of which participate in the observed variability of CPS structures (Karlyshev et al., 2005). Despite the high level of overall variability in region 2, some genes are highly conserved. These include genes for synthesis of heptose ( $h d d C, g m h A, h d d A)$ and deoxyheptose $(d m h A)$ as well of genes involves in MeOPN biosynthesis. McNally et al. (2007) showed that four genes (CJ1415c-1418c) are required for MeOPN synthesis in NCTC 11168, and these genes are highly conserved in most strains of C. jejuni. Two other genes, Cj1419c and Cj1420c, annotated as methyl transferases, are always found adjacent to the MeOPN synthesis genes, although a function has not been ascribed to them. Figure 2 indicates that $61.5 \%$ (8/13) of published CPS loci contain homologs of $\mathrm{Cj} 1415 \mathrm{c}-\mathrm{Cj} 1420 \mathrm{c}$. BLAST analysis reveals the presence of these genes not just in C. jejuni strains, but also in other

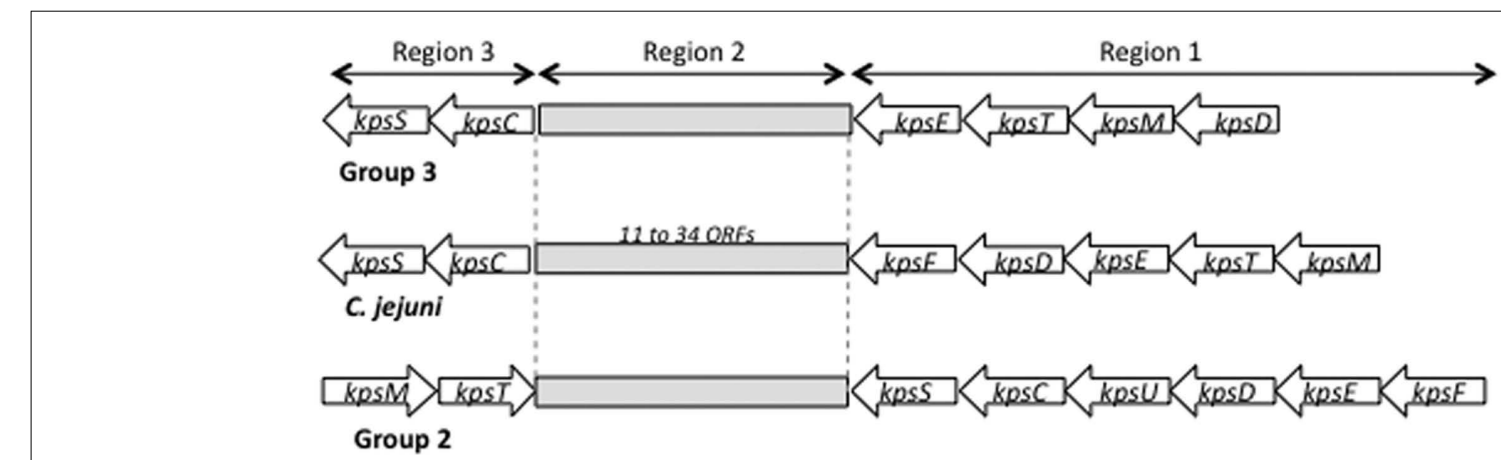

FIGURE 2 | Schematic of the capsule regions of $E$. coli group 2 and group 3 capsules compared to $C$. jejuni. 


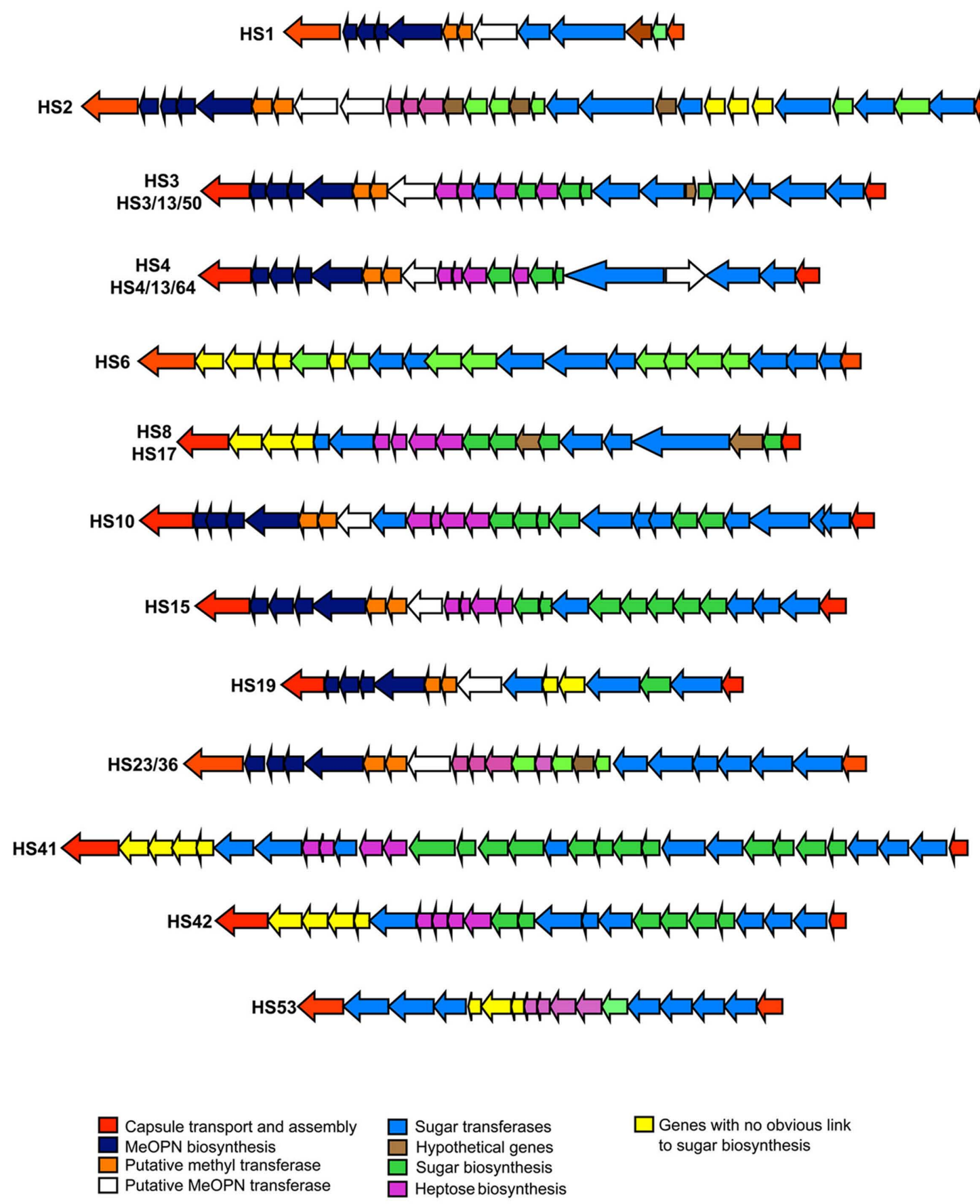

FIGURE 3 | Schematic of variable region 2 CPS loci from sequenced representative Penner serotype loci. The function or putative function of genes is color-coded, as indicated.

epsilon proteobacteria, including other species of Campylobacter, Wolinella succinogenes, and Helicobacter spp. other than H. pylori. Production of CPS in those strains in uncertain, but the high degree of protein identity and gene order of the homologs suggests a common source of origin (lateral transfer) of those genes as well as a putative biosynthesis of MeOPN by a wider number of species than originally thought (McNally et al., 2007).
McNally et al. (2007) also identified two genes adjacent to the MeOPN gene cluster, $\mathrm{Cj} 1421 \mathrm{c}$ and $\mathrm{Cj} 1422 \mathrm{c}$, as transferases responsible for addition of MeOPN to two distinct sites in the CPS of an HS2 CPS (the C-3 of $\beta$-D-GalfNAc and to the C-4 of D-glycero$\alpha$-L-gluco-heptopyranose residues). Based on similarity to these MeOPN transferases, other putative MeOPN transferases can be identified in additional C. jejuni CPS loci, all of which appear 
to be adjacent to the MeOPN biosynthesis genes. These putative MeOPN transferases contain a conserved $\mathrm{N}$-terminal region sequence and a variable $\mathrm{C}$-terminal region. The conserved region likely encodes the region of the enzyme that recognizes MeOPN, while the variable region likely reflects the different sugar residues to which MeOPN is attached.

The genes encoding enzymes for biosynthesis of heptose and deoxyheptose are also highly conserved in various CPS loci. CPS heptoses are synthesized by the products of $h d d C$ (putative D-glycero-D-manno-heptose 1-phosphate guanosyltransferase), gmhA2 (phosphoheptose isomerase), hddA (putative D-glycero-Dmanno-heptose 7-phosphate kinase). Biosynthesis of deoxyheptose has been demonstrated in C. jejuni (Karlyshev et al., 2005) and linked by homology of sequence to $d m h A$ a putative GDPmannose 4,6-dehydratase involves in the conversion of heptose to deoxyheptose. The enzymatic properties of DmhA from strain 81 to 176 were recently characterized (McCallum et al., 2011).

\section{REGULATION OF CPS EXPRESSION}

The ability of C. jejuni to undergo phase variations by slip strand mispairing during replication at homopolymeric tracts of bases is well established (Linton et al., 2000; Guerry et al., 2001; Hendrixson, 2006). C. jejuni CPS expression undergoes phase variable changes at two levels. Expression of CPS undergoes an on/off phase variation, likely due to slip strand mismatching in one or more genes in region 1 and 3 (Bacon et al., 2001; Guerry and Szymanski, 2008). Other phase variations can modulate the CPS structure. Thus, GC tracts are commonly found in $d m h A, \mathrm{Cj} 1420 \mathrm{c}$ (one of the genes adjacent to the MeOPN genes, see above), as well as putative MeOPN transferases. Thus, phase variable changes in $d m h A$ may regulate the amount of deoxyheptoses in the capsules of HS23/36 complex, and changes in the MeOPN transferases are thought to be responsible for the non-stoichiometric amounts of this modification on the CPS.

There is also some evidence that CPS expression is regulated transcriptionally. Experimental determination of the transcriptional patterns of the capsule genes has not been reported, but in silico studies by Petersen et al. (2003) suggested a rpoD $\left(\right.$ sigma $\left.^{70}\right)$-regulated operon that started with $k p s \mathrm{M}$ and extended into region 2. Transcriptional profiling studies have suggested that some of the genes involved in CPS biosynthesis are regulated differentially. Thus, four genes in region 2 were reported to be up-regulated under growth conditions with high iron (Palyada et al., 2004), and some CPS genes in both region 2 and region 3 were down regulated in vivo in the stomach of pigs (Reid et al., 2008). In vitro studies showed that four genes involved in CPS transport and three genes involved in polysaccharide biosynthesis were down regulated when $C$. jejuni was grown in the presence of HCT- 8 human intestinal epithelial cells (Corcionivoschi et al., 2009). The significance of these changes to capsule structure and virulence remain to be determined.

\section{ROLE OF CAPSULES IN VIRULENCE}

The ability to turn CPS expression on and off suggests that CPS expression may be advantageous at some points during the $C$. jejuni lifestyle and disadvantageous at others, suggesting a role in virulence. Similarly, the ability to phase vary the structure of the CPS, such as modifying the levels of MeOPN, likely serves a biological purpose. Polysaccharide capsules are important in virulence for virtually all bacteria that express these structures, but surprisingly little is understood about the role that CPS plays in $C$. jejuni-mediated disease. Bacon et al. (2001) showed that a kpsM mutant of strain 81-176 showed a modest (about 10-fold) reduction in invasion of intestinal epithelial cells in vitro. Similar results were obtained using non-encapsulated mutants of other strains by Bachtiar et al. (2007) and Corcionivoschi et al. (2009). The latter study also demonstrated that capsule expression by both $81-176$ and NCTC 11168 was reduced by passage with HCT- 8 intestinal cells in culture, as mentioned above. This result was somewhat unexpected, but the passaged cells showed reduced invasion that correlated with reduced capsule expression. However, the passaged cells represented those not attached to the monolayer; the authors did not examine capsule expression on adhered or invaded cells.

There have been a few studies that have examined the interaction of the C. jejuni CPS with various components of the host innate immune response. C. jejuni shows levels of resistance to complement killing that are comparable to those of other mucosal pathogens, and, like other mucosal pathogens, the CPS contributes to resistance to complement killing (Bacon et al., 2001; Keo et al., 2011). However, it is not clear if complement resistance is required for intestinal colonization or after invasion of the intestinal epithelium. Zilbauer et al. (2005) showed that C. jejuni 81-176 and NCTC 11168 induced human $\beta$-defensins 2 and 3 (hBD2 and hBD3) from intestinal epithelial cells in culture, but that both wildtype and $k p s M$ mutants of both strains were equally sensitive to the action of these $\beta$-defensins. This is consistent with work of Keo et al. (2011) who showed that the LOS core played a role in resistance to $\beta$-defensins and polymyxin, but that the CPS played a limited role. In contrast, capsules on other mucosal pathogens have been shown to protect against $\beta$-defensins (Campos et al., 2004). Rose et al. (2011) showed that non-encapsulated mutants showed increased production of IL-6, IL-10, and TNF-alpha from murine dendritic cells. A similar effect was seen with mutant that lacked MeOPN on the capsule, suggesting an important biological role for this unusual modification.

There are limited small animal models for C. jejuni disease. Champion et al. (2010) have developed a model using the larvae of the wax moth, Galleria mellonella. Injection of three different strains of $C$. jejuni into the larvae resulted in rapid killing. Using specific mutants the authors showed that the polysaccharide CPS of strain NCTC 11168, and specifically, the MeOPN modification, was responsible for this lethality. This may be related to the fact the MeOPN is structurally related to organophosphorus pesticides, but the significance of this finding to human disease remains to be determined. Chickens, which can become colonized with C. jejuni without disease, have been used to study commensalism. Two groups have shown that encapsulated strains colonize chickens better than unencapsulated mutants (Grant et al., 2005; Bachtiar et al., 2007). Unfortunately, there are few diarrhea models of disease for C. jejuni, but Bacon et al. (2001) showed that a kpsM mutant of 81-176 was attenuated in diarrheal disease in an infant ferret model. More convincing evidence of a role in diarrheal disease has come from vaccination studies in non-human primates (see below). 


\section{THE BURDEN OF C. JEJUNI DISEASE}

Campylobacter jejuni infection, which occurs through exposure to contaminated food and water, is a major global health problem. In the developing world it is estimated that $40-60 \%$ of children under the age 5 will develop at least one symptomatic infection, usually occurring during the first year of life (reviewed in Coker et al., 2002). Epidemiologic studies are lacking in geographically diverse settings, but those that do exist suggest that repeated pediatric infections are not uncommon. Recent data suggest that Campylobacter may be even more important than previously thought. The Global Enterics Multi-Center Study (GEMS), a prospective, multi-center, case-control study of acute diarrhea in children 0-59 months of age funded by the Bill and Melinda Gates Foundation, has found in 2-year follow-up data that Campylobacter infections in Asia (Bangladesh, India, Pakistan) represent important enteric infections leading to severe diarrhea in the first years of life (Levine, 2011). A recent passive clinical surveillance study in a defined catchment population from Karachi, Pakistan reports an annual incidence of C. jejuni infection of 29 per 1,000 personyears, with peak incidence at around 2 years of age (Soofi et al., 2011). It is notable that $C$. jejuni infections were more frequently diagnosed than Shigella, though Shigella was more often associated with dysentery. Additionally, for the developing world, one must consider the association between Campylobacter and HIV infection where incidence may be increased, and morbidity and mortality are found to be higher among HIV positive individuals (Sorvillo et al., 1991; Molina et al., 1995; Tee and Mijch, 1998). In a case series of 38 patients with HIV and Campylobacter infection, acute diarrhea, fever, and abdominal pain was a predominant symptom complex, however $11 \%$ had bacteremia, and $8 \%$ experienced chronic diarrhea (Molina et al., 1995). Furthermore, long-term carriage of Campylobacter can occur, sometimes associated with recurrent attacks of enteritis and bacteremia among HIV infected individuals. Given the growing HIV pandemic, it is estimated that the burden of campylobacteriosis in developing countries may be among the top 10 in 2020.

In the industrialized world, general population incidence estimates based on passive surveillance vary depending on geography and over time with rates in the US averaging around 15/100,000 with a slow decline over the past decade, and rates in Europe around 50-90/100,000 with rising trends (reviewed in Janssen et al., 2008). Some countries in Eastern Europe and New Zealand have higher incidence rates of 300-400/100,000 per year. Due to underreporting of these types of illness, true rates of campylobacteriosis are considered to be 10-100 times higher than those reported historically. To this end, recent population-based studies in a number of developed countries have employed varied study designs to estimate overall incidence of Campylobacter infections through use of cohort studies, state of the art and standardized identification methods, surveys, and active surveillance networks (Table 1). These studies confirm the underreporting and case ascertainment bias associated with passive surveillance studies and find that incidence of Campylobacter infection ranges from 3 to 15 cases per 1,000 person-years.

Finally, travelers represent unique populations that are at high risk for Campylobacter, where globally it occurs in 5-15\% of diarrheal cases (Riddle et al., 2006; Shah et al., 2009). Campylobacter infections are considerably more frequent in some areas of SE Asia. Ravel et al. (2011) recently reported data from an enhanced, passive surveillance system of travel-related diseases caused by enteropathogens within a Canadian community from June 2005 to May 2009. Of the 446 cases of travel-related disease

Table 1 | Global estimates of Campylobacter incidence in developed countries.

\begin{tabular}{|c|c|c|c|c|c|}
\hline Reference & Kubota et al. (2008) & Tam et al. (2012) & De Wit et al. (2001) & Hall et al. (2008) & Scallan et al. (2011) \\
\hline Country & Japan & UK & Netherlands & Australia & US \\
\hline Year of study & 2006-2007 & 2008-2009 & 1998-1999 & 2000-2004 & 2006 \\
\hline Study design & $\begin{array}{l}\text { Two 2-week } \\
\text { cross-sectional, } \\
\text { population-based } \\
\text { telephone surveys } \\
\text { combined with } \\
\text { catchment area } \\
\text { surveillance }\end{array}$ & $\begin{array}{l}\text { Prospective, } \\
\text { community cohort } \\
\text { study and prospective } \\
\text { study of general } \\
\text { practice presentation in } \\
\text { national surveillance } \\
\text { system }\end{array}$ & $\begin{array}{l}\text { Prospective } \\
\text { population-based } \\
\text { study with nested } \\
\text { case-control } \\
\text { study in general } \\
\text { population }\end{array}$ & $\begin{array}{l}\text { Empirical model } \\
\text { based on published } \\
\text { and unpublished } \\
\text { data from multiple } \\
\text { active/passive } \\
\text { surveillance sources }\end{array}$ & $\begin{array}{l}\text { Empirical model based } \\
\text { on published and unpub- } \\
\text { lished data from mul- } \\
\text { tiple active/passive sur- } \\
\text { veillance sources }\end{array}$ \\
\hline Numbers & $\begin{array}{l}\text { 4,247 Household } \\
\text { interviews, } 8,462 \\
\text { laboratory confirmed } \\
\text { cases ascertained in } \\
\text { active surveillance }\end{array}$ & $\begin{array}{l}\text { 6,836 Cohort } \\
\text { participants, } 800,000 \\
\text { catchment area for } \\
\text { national surveillance }\end{array}$ & $\begin{array}{l}\text { 4,860 Patients } \\
\text { enrolled in cohort }\end{array}$ & Not applicable & Not applicable \\
\hline $\begin{array}{l}\text { Incidence } \\
\text { estimate }(95 \% \\
\mathrm{Cl}), \text { per 1,000 } \\
\text { person-years }\end{array}$ & $15.1(7.4-28.6)$ & $10.9(7.4-15.9)$ & $4.8(1.7-10.4)$ & $11.8(7.6-26.7)$ & 2.8 (not given) \\
\hline $\begin{array}{l}\text { Foodborne illness } \\
\text { rank }\end{array}$ & 1 of 3 overall & $\begin{array}{l}4 \text { of } 12 \text { overall; } 1 \text { of } 5 \\
\text { bacterial }\end{array}$ & 1 of 5 bacterial & 1 of 3 overall & $\begin{array}{l}4 \text { of } 31 \text { overall; } 3 \text { of } 21 \\
\text { bacterial }\end{array}$ \\
\hline
\end{tabular}


due to enteropathogens reported, Campylobacter was the most frequently identified cause $(n=123,28 \%)$. Similarly, in a report from the GeoSentinel Travel Network, a clinician-based sentinel surveillance data for 17,353 ill returned travelers, Campylobacter was identified in 8.5 per 100 diarrheal cases and was the leading bacterial etiology (Freedman et al., 2006).

Taken together, these data highlight the clear importance of Campylobacter in global populations in terms of disease incidence, but do not completely portray the enormity of Campylobacter as a public health problem. An increasing number of studies are highlighting Campylobacter-associated chronic health sequelae (Table 2). Beyond the well described association with GuillainBarré Syndrome (GBS), the leading cause of acute flaccid paralysis in the developing world for which up to a third can be attributed to Campylobacter, reactive arthritis, inflammatory bowel disease (particularly Crohn's Disease), and irritable bowel syndrome are also recognized as post-campylobacteriosis sequelae in industrialized populations, although there is lack of information on these sequelae in the developing world. Furthermore, it will be of interest to explore possible associations with CPS type as well as other virulent mechanisms and these health sequelae.

From a public health perspective, some country-level approaches have been attempted to quantify and characterize the burden and cost associated with acute diarrheal infections and their chronic consequences. For example, in New Zealand it is estimated that major foodborne illness and its chronic health consequences costs society approximately $\$ 86$ million per year, and $90 \%$ of this cost is attributed to lost productivity due to absence from work (Lake et al., 2010), and campylobacteriosis is shown to account for approximately $90 \%$ of the estimated cost of foodborne illness. Cost and burden of disease studies in Australia and the Netherlands have also been conducted outlining substantial individual and societal costs in which acute disease is either matched or exceeded by the chronic consequences (Mangen et al., 2004). Beyond the country-level perspective focusing on domestically acquired foodborne infections, populations at high risk for acute infectious diarrhea (e.g., travelers, military) should also be considered in estimating the full burden of disease and the potential value of current and novel interventions. It should be noted, while not as well studied, the chronic consequences of Campylobacter infections in developing world populations should not be underestimated. Given the known mucosal barrier disruption effects of Campylobacter infection (Beltinger et al., 2008), the contribution of this particular infection may emerge as an important factor to malnutrition and associated consequences in the developing world. An international group of investigators are collaborating on a project entitled The Interactions of Malnutrition and Enteric Infections (MAL-ED): Consequences for Child Health and Development (http://mal-ed.fnih.org/), involving a network of eight sites that are exploring the factors associated with a child's risk of enteric infection, chronic diarrhea, malnutrition, as well with impaired gut function, vaccine response, and cognitive and physical development (Lorntz et al., 2006; Tarleton et al., 2006; Santos et al., 2008; Oria et al., 2010). Given the known mucosal barrier disruption effects of Campylobacter infection, it will be important to follow.

While more study utilizing improved diagnostics and characterizing the pathogenesis of acute and chronic health effects of Campylobacter infections is needed, the data that is available suggest that control of Campylobacter should be considered a global public health priority. Promotion and strengthening of food safety systems, good manufacturing practices and educating retailers and consumers about appropriate food handling and avoiding contamination are needed now and can be employed. Education of consumers and training of food handlers in safe food handling is one of the most critical interventions in the prevention of foodborne illnesses. Due to the challenges of implementing these aforementioned strategies, however, vaccines for use in travelers, military personnel, and pediatric populations in the developing world are much needed. To this end, we have explored the feasibility of CPS conjugate vaccines against $C$. jejuni.

\section{CPS CONJUGATE VACCINES AS A STRATEGY AGAINST C. JEJUNI}

CPS-based vaccine strategies have been very successful at reducing the overall disease incidence of several encapsulated bacteria including Streptococcus pneumoniae, N. meningitidis, and $H$. influenzae (reviewed in Lesinski and Westernick, 2001a; Knuf et al.,

Table 2 | Summary evidence of post-Campylobacter infection risk of select chronic health consequences.

\begin{tabular}{|c|c|c|c|}
\hline Sequelae & $\begin{array}{l}\text { Post-infective } \\
\text { attributable risk* }\end{array}$ & Comment & Reference \\
\hline Guillain Barré syndrome & 1 per 1,000 & $14-32 \%$ of GBS cases can be attributed to $C$. jejuni & Nachamkin et al. (2000) \\
\hline Reactive arthritis & $1-5 \%$ & $5 \%$ of $C$. jejuni ReA may be chronic or relapsing & Pope et al. (2007) \\
\hline $\begin{array}{l}\text { Inflammatory bowel } \\
\text { disease }\end{array}$ & $3-4$ per 10,000 & $\begin{array}{l}\text { Recent evidence suggests that } C \text {. jejuni can breach the intestinal } \\
\text { barrier and may prime the intestine for chronic inflammatory } \\
\text { responses in susceptible individuals (Kalischuk and Buret, 2010) }\end{array}$ & $\begin{array}{l}\text { Gradel et al. (2009), Jess et al. } \\
\text { (2011), Rodriguez et al. (2006) }\end{array}$ \\
\hline $\begin{array}{l}\text { Irritable bowel } \\
\text { syndrome }\end{array}$ & $1-10 \%$ & $\begin{array}{l}\text { IBS developed in } 36 \% \text { of patients associated with a large } \\
\text { waterborne outbreak of mixed Campylobacter and STEC in } \\
\text { Walkerton, Canada. Symptoms persist in approximately } 40-50 \% \\
\text { at } 5-7 \text { years }\end{array}$ & $\begin{array}{l}\text { Rodriguez and Ruigomez (1999), } \\
\text { Thornley et al. (2001), Dunlop et al. } \\
\text { (2003), Marshall et al. (2006) }\end{array}$ \\
\hline
\end{tabular}

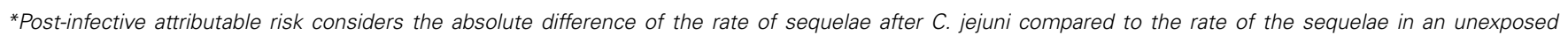
population. 
2011). Given the success of these capsular-based vaccines, we have begun studies to determine the feasibility of a capsule conjugate vaccine approach to prevent disease caused by C. jejuni.

Most capsular polysaccharides are thymus-independent (TI) antigens meaning that helper $\mathrm{T}$ cells are needed to generate robust, long-lived antibody responses. Although adults can generate some antibody responses to purified capsules administered as a vaccine (Lesinski and Westernick, 2001b), children $<2$ years of age do not develop strong responses to TI antigens (Rijkers et al., 1998). As most carbohydrate antigens cannot directly activate naïve $\mathrm{T}$ cells, a carrier protein is often included to optimize immune responses (Knuf et al., 2011). This vaccine strategy effectively converts a TI antigen into a thymus dependent (TD) antigen that allows boosting of the immune response, IgG antibody class switching, and the generation of memory cells possessing antibody with higher avidity for CPS (Lesinski and Westernick, 2001b).

In the initial studies, CPS was purified from C. jejuni strains 81176 (HS23/36) and CG8486 (HS4 complex) and conjugated to the carrier protein, $\mathrm{CRM}_{197}$, which is a mutant diphtheria toxin subunit, using reductive amination (Monteiro et al., 2009). BALB/c mice were immunized subcutaneously with escalating doses of C. jejuni CPS-CRM 197 . Maximal titers were generated following three doses of vaccine. One month after the last dose of vaccine, immunized mice were challenged with each respective strain using an intranasal infection model to determine if vaccination conferred protection. Immunized mice exhibited significantly lower levels of sickness following challenge suggesting that antibodies generated against the capsule afforded some protection against infection with C. jejuni in this model. However, testing in a more relevant model was still required.

The New World monkey, Aotus nancymaae, has been shown to develop diarrheal disease that mimics aspects of human illness following orogastric challenge with C. jejuni (Jones et al., 2006). To determine if immunization with CPS-CRM 197 could prevent diarrheal disease, monkeys were immunized with $2.5 \mu \mathrm{g}$ of polysaccharide adjuvanted with alum three times with an interval of 6 weeks between subcutaneous injections. Nine weeks following the third immunization, monkeys were challenge orogastrically with approximately $10^{11} \mathrm{CFU}$ of $C$. jejuni $81-176$. The diarrheal attack rate for non-immunized control monkeys was 70\%. However, $100 \%$ of vaccinated animals (14/14) were protected against diarrheal disease (Monteiro et al., 2009). This work was the first demonstration that a capsule conjugate vaccine could protect against enteric disease and also suggests that the capsule plays a critical role in virulence.

These proof-of-concept studies proved the feasibility of using a capsule conjugate vaccine to prevent illness caused by C. jejuni. However, before developing a vaccine for practical use, the number of CPS types needed to achieve broad coverage against the most prevalent strains of $C$. jejuni needs to be determined

\section{CPS TYPING BY MULTIPLEX PCR}

The likely target population for a vaccine against $C$. jejuni would be civilian and military travelers to endemic regions and pediatric populations living in endemic areas. However, while there are extensive data on Penner types of $C$. jejuni strains from the developed world, particularly the Europe and Canada, there are limited data from less developed countries (LDC) where the incidence of C. jejuni diarrhea is high. Figure 4 summarizes published data on Penner types from sporadic clinical cases based on $>16,000$ clinical isolates from the developed world and about 700 from LDC. A non-systemic review of Penner typing for sporadic clinical cases identified HS4, HS2, and HS1 as the most common globally. The HS4 complex was the most prevalent, accounting for 21.2 and $13.2 \%$ of all cases for the developed and LDC, respectively, followed by HS2 (16.4 and 7.96\% for developed and LDC, respectively) and the HS1 complex (10.7 and $6.81 \%$ for developed and LDC, respectively). Penner types HS3, HS5, and HS8 were also prevalent worldwide, while other serotypes tend to show geographic and population-based variability. More than $10 \%$ of isolates from the developed world and $>21 \%$ from the developing world were non-typeable in the Penner scheme. This could reflect the existence of CPS types not covered in the Penner typing scheme, but it is also in partly due to the fact that Penner serotyping requires CPS expression and a certain percentage of these isolates have turned capsule expression off by phase variation (Poly, unpublished).

To overcome the limited data on CPS distribution in LDC's, a C. jejuni CPS multiplex-based PCR was recently developed and validated. The current CPS multiplex is composed of a total of 14 primer pairs, separated into two mixes, alpha and beta (Poly et al., 2011). Primers were designed on specific sequences of available $C$. jejuni class 2 loci (Figure 2). Most of the Penner serotypes that fall into related complexes are recognized with the same pair of primers. Thus, the multiplex system does not distinguish between HS23 and HS36 and other similar complexes of Penner types, which have very similar CPS structures that crossreact immunologically. Attribution of CPS type is deciphered based on the length of the PCR product after electrophoresis on a $2 \%$ agarose gel along with a molecular weight ladder. This technology was validated on a total of 244 strains of known serotype and shown to have sensitivities and specificities ranging from 90 to $100 \%$. A perfect correlation between CPS type and Penner type was demonstrated. This technology is still expanding and is currently being evaluated in multiple laboratories worldwide. One advantage of the multiplex system over classical serotyping is that the multiplex is independent of phase variation in capsule expression. Collection of additional data by the multiplex PCR will provide a more accurate picture of C. jejuni CPS distribution, particularly in LDCs, that will dictate the final formulation of an effective CPS conjugate vaccine. The ability to rapidly determine capsule type will also facilitate studies to determine if specific CPS types are associated with more severe acute diarrheal disease and with the chronic sequelae, particularly functional bowel disorders.

\section{ACKNOWLEDGMENTS}

Work at NMRC was funded by NavyWork Unit 6000.RAD1.DA3. A0308 and work at University of Guelph was funded by NSERC. The views expressed in this manuscript are those of the authors and do not necessarily reflect the official policy or position of the Department of the Navy, Department of Defense, nor the U. S. Government. Patricia Guerry is an employe of the U. S. government and Mark Riddle is a military service member. This work was 


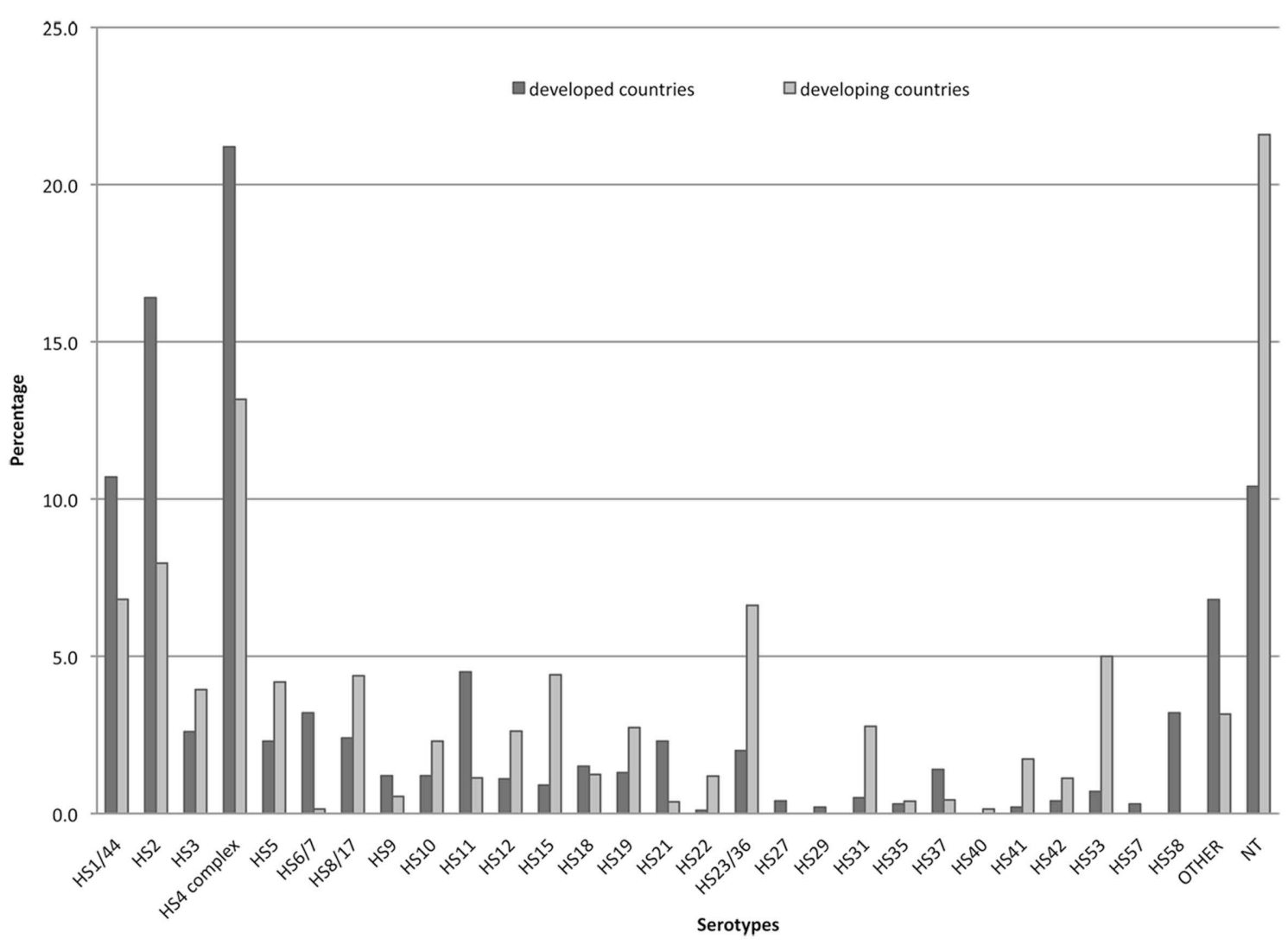

FIGURE 4 | Penner serotype distribution worldwide. The data represent a total of $>16,000$ strains from developed countries (Penner and Hennessy, 1980; McMyne et al., 1982; Penner et al., 1983; Jones et al., 1985; Patton et al., 1985; Mills et al., 1991; Albert et al., 1992; Skirrow et al., 1993; Marshall et al., 1994; Owen et al., 1994; Nielsen et al., 1997, 2006; Frost et al., 1998; McKay et al., 2001; Oza et al., 2002; Wareing et al., 2002; Woodward and
Rodgers, 2002; Karenlampi et al., 2003; Siemer et al., 2004; Cornelius et al., 2005; Fussing et al., 2007; McTavish et al., 2008); and far fewer strains (711) from less developed countries, including Central Africa (Georges-Courbot et al., 1989), South Africa (Lastovica et al., 1986), South Africa (Lastovica et al., 1986), Egypt (Poly et al., 2011), Bangladesh (Neogi and Shahid, 1987), Kuwait (Sjogren et al., 1989), and Thailand (Poly et al., 2011).

prepared as part of their official duties. Title 17 USC 105 provides that "Copyright protection under this title is not available for any work of the United States Government." Title USC 101 defines

\section{REFERENCES}

Albert, M. J., Leach, A., Asche, V., Hennessy, J., and Penner, J. L. (1992). Serotype distribution of Campylobacter jejuni and Campylobacter coli isolated from hospitalized patients with diarrhea in central Australia. J. Clin. Microbiol. 30, 207-210.

Aspinall, G. O. (1998). Lipopolysaccharides and associated carbohydrate polymers from Campylobacter jejuni and Helicobacter pylori. Carbohydr. Eur. 21, 24-29.

Aspinall, G. O., McDonald, A. G., and Pang, H. (1992). Structures of the $\mathrm{O}$-chains from lipopolysaccharides of Campylobacter jejuni serotypes O:23 and O:36. Carbohydr. Res. 231, 13-30.

Aspinall, G. O., McDonald, A. G., Pang, H., Kurjanczyk, L. A., and Penner,
J. L. (1993). An antigenic polysaccharide from Campylobacter coli serotype O:30. Structure of a teichoic acid-like antigenic polysaccharide with the lipopolysaccharide. $J$. Biol. Chem. 268, 18321-18329.

Aspinall, G. O., Monteiro, M. A., Pang, H., Kurjanczyk, L. A., and Penner, J. L. (1995a). Lipooligosaccharide of Campylobacter lari strain PC637. Structure of the liberated oligosaccharide and an associated extracellular polysaccharide. Carbohydr. Res. 279, 227-244.

Aspinall, G. O., Monteiro, M. A., and Pang, H. (1995b). Lipooligosaccharide of Campylobacter lari type strain ATCC 35221. Structure of the liberated oligosaccharide and an associated extracellular polysaccharide. Carbohydr. Res. 279, 245-264.

a U.S. government work as a work prepared by a military service member or employe of the U.S. government as part of that person's official duties.

Aspinall, G. O., Lynch, C. M., Pang, H., Shaver, R. T., and Moran, A. P. (1995c). Chemical structures of the core region of Campylobacter jejuni O:3 lipopolysaccharide and an associated polysaccharide. Eur. J. Biochem. 231, 570-578.

Bachtiar, B. M., Coloe, P. J., and Fry, B. N. (2007). Knockout mutagenesis of the kpsE gene of Campylobacter jejuni 81116 and its involvement in bacterium-host interactions. FEMS Immunol. Med. Microbiol. 49, 149-154.

Bacon, D. J., Szymanski, C. M., Burr, D. B., Silver, R. P., Alm, R. A., and Guerry, P. (2001). A phase variable capsule is involved in virulence of Campylobacter jejuni 81-176. Mol. Microbiol. 40, 769-777.

Beltinger, J., Del Buono, J., Skelly, M. M., Thornley, J., Spiller, R. C., Stack, W.
A., and Hawkey, C. J. (2008). Disruption of colonic barrier function and induction of mediator release by strains of Campylobacter jejuni that invade epithelial cells. World J. Gastroenterol. 14, 7345-7352.

Campos, M. A., Vargas, M. A., Regueiro, V., Llompart, C. M., Alberti, S., and Bengoechae, J. A. (2004). Capsule polysaccharide mediates bacterial resistance to antimicrobial peptides. Infect. Immun. 72, 7107-7114.

Champion, O. L., Karlyshev, A. V., Senior, N. J., Woodward, M., La Ragione, R., Howard, S. L., Wren, B. W., and Titball, R. W. (2010). Insect infection model for Campylobacter jejuni reveals that $\mathrm{O}$ methyl phosphoramidate has insecticidal activity. J. Infect. Dis. 201, 776-782. 
Chen, Y. H., Poly, F., Pakulski, Z., Guerry, P., and Monteiro, M. A. (2008). The chemical structure and genetic locus of the Campylobacter jejuni CG8486 (HS4) capsular polysaccharide: the identification of 6-deoxy-D-idoheptopyranose. Carbohydr. Res. 343, 1034-1040.

Coker, A. O., Isokpehi, R. D., Thomas, B. N., Amisu, K. O., and Obi, C. L. (2002). Human campylobacteriosis in developing countries. Emerging Infect. Dis. 8, 237-244.

Corcionivoschi, N., Clyne, M., Lyons, A., Elmi, A., Gundogdu, O., Wren, B. W., Dorrell, N., Karlyshev, A. V., and Bourke, B. (2009). Campylobacter jejuni cocultured with epithelial cells reduces surface capsular polysaccharide expression. Infect. Immun. 77, 1959-1967.

Corcoran, A. T., Annuk, H., and Moran, A. P. (2006). The structure of the lipid anchor of Campylobacter jejuni polysaccharide. FEMS Microbiol. Lett. 257, 228-235.

Cornelius, A. J., Nicol, C., and Hudson, J. A. (2005). Campylobacter spp. in New Zealand raw sheep liver and human campylobacteriosis cases. Int. J. Food Microbiol. 99, 99-105.

De Wit, M. A. S., Koopmans, M. P. G., Kortbeek, L. M., Wannet, W. J. B., Vinje, J., Van Leusden, F., Bartelds, A. I. M., and van Duynhoven, Y. T. (2001). Sensor, a population-based cohort study on gastroenteritis in the Netherlands: incidence and etiology. Am. J. Epidemiol. 154, 666-674.

Dunlop, S. P, Jenkins, D., Neal, K. R., and Spiller, R. C. (2003). Relative importance of enterochromaffin cell hyperplasia, anxiety, and depression in postinfectious IBS. Gastroenterology 125, 1651-1659.

Fouts, D. E., Mongodin, E. F., Mandrell, R. E., Miller, W. G., Rasko, D. A., Ravel, J., Brinkac, L. M., DeBoy, R. T., Parker, C. T., Dougherty, S. C., Dodson, R. J., Durkin, A. S., Madupu, R., Sullivan, S. A., Shetty, J. U., Ayodeji, M. A., Shvartsbeyn, A., Schatz, M. C., Badger, J. H., Fraser, C. M., and Nelson, K. E. . (2005). Major structural differences and novel potential virulence mechanisms from the genomes of multiple Campylobacter species. PLoS Biol. 3, e15. doi:10.1371/journal.pbio.0030015

Freedman, D. O., Weld, L. H., Kozarsky, P. E., Fisk, T., Robins, R., and von Sonnenburg, F. (2006). Spectrum of disease and relation to place of exposure among ill returned travellers. $N$. Engl. J. Med. 354, 119-130.

Frost, J. A., Oxa, A. N., Thwaites, R. T., and Rowe, B. (1998). Serotyping scheme for Campylobacter jejuni and Campylobacter coli based on direct agglutination of heat-stable antigens. J. Clin. Microbiol. 36, 335-339.

Fussing, V., Moller, N. E., Neimann, J., and Engberg, J. (2007). Systematic serotyping and riboprinting of Campylobacter spp. improves surveillance: experiences from two Danish counties. Clin. Microbiol. Infect. 13, 635-642.

Georges-Courbot, M. C., Gouandjika, I., Martin, P. M., and Georges, A. J. (1989). Biotype and Lior serogroup distribution of enteric Campylobacter isolated from children in Bangui (Central African Republic), and comparison with Penner serotypes. Res. Microbiol. 140, 489-497.

Gilbert, M., Mandrell, R. E., Parker, C. T., Li, J., and Vinogradov, E. (2007). Structural analysis of the capsular polysaccharide from Campylobacter jejuni RM1221. Chembiochem. 8, 625-631.

Gradel, K. O., Nielsen, H. L., Schønheyder, H. C., Ejlertsen, T., Kristensen, B., and Nielsen, H. (2009). Increased short- and long-term risk of inflammatory bowel disease after Salmonella or Campylobacter gastroenteritis. Gastroenterology 137, 495-501.

Grant, A. J., Coward, C., Jones, M. A., Woodall, C. A., Barrow, P. A., and Maskell, D. J. (2005). Signature-tagged transposon mutagenesis studies demonstrate the dynamic nature of cecal colonization of 2-week old chickens by Campylobacter jejuni. Appl. Environ. Microbiol. 71, 8031-8041.

Guerry, P., and Szymanski, C. M. (2008). Campylobacter sugars sticking out. Trends Microbiol. 16, 428-435.

Guerry, P., Szymanski, C. M., Prendergast, M. M., Hickey, T. E., Ewing, C. P., Pattarini, D. L., and Moran, A. P. (2001). Phase variation of Campylobacter jejuni 81-176 lipooligosaccharide affects ganglioside mimicry and invasiveness in vitro. Infect. Immun. 70, 787-793.

Hall, G. Y. K., Raupach, J., Becker, N., and Kirk, M. (2008). Estimating community incidence of Salmonella, Campylobacter, and Shiga toxinproducing Escherichia coli infections, Australia. Emerging Infect. Dis. 14, 1601-1609.

Hannify, O. M., Shashkov, A. S., Moran, A. P., Prendergast, M. M., Senchenkova, S. N., Knirel, Y. A., and Savage, A. V. (1999). Chemical structure of a polysaccharide from Campylobacter jejuni 176.83 (serotype O:41) containing only furanose sugars. Carbohydr. Res. 319, 124-132.
Hendrixson, D. R. (2006). A phasevariable mechanism controlling the Campylobacter jejuni FlgR response regulator influences commensalism. Mol. Microbiol. 61, 1646-1659.

Janssen, R., Krogfelt, K. A., Cawthraw, S. A., van Pelt, W., Wagenaar, J. A., and Owen, R. J. (2008). Host-pathogen interactions in Campylobacter infections: the host perspective. Clin. Microbiol. Rev. 21, 505-518.

Jess, T., Simonsen, J., Nielsen, N. M., Jørgensen, K. T., Bager, P., Ethelberg, S. and Frisch, M. (2011). Enteric Salmonella or Campylobacter infections and the risk of inflammatory bowel disease. Gut 60, 318-324.

Jones, D. M., Sutcliffe, E. M., and Abbott, J. D. (1985). Serotyping of Campylobacter species by combined use of two methods. Eur. J. Clin. Microbiol. 4, 562-565.

Jones, F. R., Baqar, S., Gozalo, A., Nunez, G., Espinoza, N., Reyes, S. M., Salazar, M., Meza, R., Porter, C. K., and Walz, S. E. (2006) New World monkey, Aotus nancymaae as a model for Campylobacter jejuni infection and immunity. Infect. Immun. 74, 790-793.

Kalischuk, L. D., and Buret, A. G. (2010). A role for Campylobacter jejuni-induced enteritis in inflammatory bowel disease? Am. J. Physiol. Gastrointest. Liver Physiol. 298, G1-G9.

Kanipes, M. I., Akelatis, A., Guerry, P., and Monteiro, M. A. (2006). Mutation of waaC encoding heptosyl transferase I in Campylobacter jejuni 81-176 affects the structure of both lipooligosaccharide and capsular carbohydrate. J. Bacteriol. 188, 3273-3279.

Karenlampi, R., Rautelin, H., Hakkinen, M., and Hanninen, M. L. (2003). Temporal and geographical distribution and overlap of Penner heat-stable serotypes and pulsedfield gel electrophoresis genotypes of Campylobacter jejuni isolates collected from humans and chickens in Finland during a seasonal peak. $J$. Clin. Microbiol. 41, 4870-4872.

Karlyshev, A. V., Champion, O. L. Churcher, C., Brisson, J.-R., Jarrell, H. C., Gilbert, M., Brochu, D. St. Michael, F., Li, J., Wakarchuk, W. W., Goodhead, I., Sanders, M., Stevens, K., White, B., Parkhill, J., Wren, B. W., and Szymanski, C. M. (2005). Analysis of Campylobacter jejuni capsular loci reveals multiple mechanisms for the generation of structural diversity and the ability to form heptoses. Mol. Microbiol. 55 , 90-103.
Karlyshev, A. V., Linton, D., Gregson, N. A., Lastovica, A. J., and Wren, B. W. (2000). Genetic and biochemical evidence of a Campylobacter jejuni capsular polysaccharide that accounts for Penner serotype specificity. Mol. Microbiol. 35, 529-541.

Keo, T., Collins, J., Kunwar, P., Blaser, M. J., and Iovine, N. M. (2011). Campylobacter capsule and lipooligosaccharide confer resistance to serum and cationic antimicrobials. Virulence 2, 30-40.

Knuf, M., Kowalzik, F., and Kieninger, D. (2011). Comparative effects of carrier proteins on vaccineinduced immune response. Vaccine 29, 4881-4890.

Kubota, K., Iwasaki, E., Inaqaki, S., Nokubo, T., Sakurai, Y., Komatsu, M., Toyofuku, H., Kasuga, F., Angulo, F. J., and Morikawa, K. (2008). The human health burden of foodborne infections caused by Campylobacter, Salmonella and Vibrio parahaemolyticus in Miyagi Prefecture, Japan. Foodborne Pathog. Dis. 5, 641-648.

Lake, R. J., Cressey, P. J., Campbell, D. M., and Oakley, E. (2010). Risk ranking for foodborne microbial hazards in New Zealand: burden of disease estimates. Risk Anal. 30, 743-752.

Lastovica, A. J., Le, R. E., Congi, R. V., and Penner, J. L. (1986). Distribution of sero-biotypes of Campylobacter jejuni and Campylobacter coli isolated from paediatric patients. J. Med. Microbiol. 21, 1-5.

Lesinski, G. B., and Westernick, M. A. (2001a). Vaccines against polysaccharide antigens. Curr. Drug Targets Infect. Disord. 1, 325-334.

Lesinski, G. B., and Westernick, M. A (2001b). Novel vaccine strategies to T-independent antigens. J. Microbiol. Methods 47, 135-149.

Levine, M. M. (2011). "Etiology and outcomes of acute moderate and severe diarrheal infections in young children in developing countries: insights from the Global Enteric Multi-Center Study (GEMS)," in 6th International Conference on Vaccines for Enteric Diseases, Cannes.

Linton, D., Gilbert, M., Hitchen, P. G., Dell, A., Morris, H. R., Wakarchuk, W. W., Gregson, N. A., and Wren, B. W. (2000). Phase variation of a b1,3 galactosyltransferase involved in generation of the ganglioside GM1like lipooligosaccharide of Campy lobacter jejuni. Mol. Microbiol. 37, 501-514.

Lorntz, B., Soares, A. M., Moore, S. R., Pinkerton, R., Gansneder, B., Bovbjerg, V. E., Guyatt, H., Lima, 
A. M., and Guerrant, R. L. (2006). Early childhood diarrhea predicts impaired school performance. Pediatr. Infect. Dis. J. 25, 513-520.

Mangen, M. J., Havelaar, A. H., and De Wit, G. A. (2004). Campylobacteriosis and Sequelae in the Netherlands-Estimating the Disease Burden and the Cost-of-Illness. RIVM report 250911, Bilthoven: National Institute for Public Health and Environment (RIVM).

Marshall, J. K., Thabane, M., Garg, A. X., Clark, W. F., Savadori, M., Collins, S. M., and Walkerton Study Investigators. (2006). Incidence and epidemiology of irritable bowel syndrome after a large waterborne outbreak of bacterial dysentery. Gastroenterology 131, 445-448.

Marshall, L. E., Boswell, T. C., and Kudesia, G. (1994). False positive legionella serology in Campylobacter infection: Campylobacter serotypes, duration of antibody response and elimination of cross-reactions in the indirect fluorescent antibody test. Epidemiol. Infect. 112, 347-357.

McCallum, M., Shaw, G. S., and Creuzenet, C. (2011). Characterization of the dehydratase WcbK and the reductase $\mathrm{WcaG}$ involved in GDP-6-deoxy-amnno-heptose biosynthesis in Campylobacter jejuni. Biochem. J. 439, 235-248.

McDonald, A. G. (1993). Lipopolysaccharides from Campylobacter. Ph. D. thesis. York University, Toronto, CA.

McKay, D., Fletcher, J., Cooper, P., and Thomson-Carter, F. M. (2001). Comparison of two methods for serotyping Campylobacter spp. J. Clin. Microbiol. 39, 1917-1921.

McMyne, P. M., Penner, J. L., Mathias, R. G., Black, W. A., and Hennessy, J. N. (1982). Serotyping of Campylobacter jejuni isolated from sporadic cases and outbreaks in British Columbia. J. Clin. Microbiol. 16, 281-285.

McNally, D. J., Jarell, H. C., Li, J., Khieu, N. H., Vinogradov, E., Szymanski, C. M., and Brisson, J. R. (2005). The HS:1 serostrain of Campylobacter jejuni has a complex teichoic acid-like capsular polysaccharide with nonstoichiometric fructofuranose branches and O-methyl phosphoramidite groups. FEBS $J$. 272, 4407-4422.

McNally, D. J., Lamoureux, M. P., Karlyshev, A. V., Fiori, L. M., Li, J., Thacker, G., Coleman, R. A., Khieu, N. H., Wren, B. W., Brisson, J. R., Jarrell, H. C., and Szymanski, C. M. (2007). Commonality and biosynthesis of the O-methyl phosphoramidate capsule modification in
Campylobacter jejuni. J. Biol. Chem. 282, 28566-28576.

McTavish, S. M., Pope, C. E., Nicol, C., Sexton, K., French, N., and Carter, P. E. (2008). Wide geographical distribution of internationally rare Campylobacter clones within New Zealand. Epidemiol. Infect. 136, 1244-1252.

Mills, S. D., Congi, R. V., Hennessy, J. N., and Penner, J. L. (1991). Evaluation of a simplified procedure for serotyping Campylobacter jejuni and Campylobacter coli which is based on the $\mathrm{O}$ antigen. J. Clin. Microbiol. 29, 2093-2098.

Molina, J. C. I., Casin, I., Hausfater, P., Giretti, E., Welker, Y., Decazes, J., Garrait, V., Lagrange, P., and Modai, J. (1995). Campylobacter infections in HIV-infected patients: clinical and bacteriological features. AIDS 9, 881-885.

Monteiro, M. A., Baqar, S., Hall, E. R., Chen, Y.-H., Porter, C. K., Bentzel, D. E., Applebee, L., and Guerry, P. (2009). A capsule polysaccharide conjugate vaccine against diarrheal disease caused by Campylobacter jejuni. Infect. Immun. 77, 1128-1136.

Muldoon, J., Shashkov, A. S., Moran, A. P., Ferris, J. A., Senchenkova, S. N., and Savage, A. V. (2002). Structures of two polysaccharides of Campylobacter jejuni 81116. Carbohydr. Res. 337, 2223-2229.

Nachamkin, I., Allos, B. M., and Ho, T. W. (2000). "Campylobacter jejuni infection and the association with Guillain-Barré syndrome," in Campylobacter, 2nd Edn, eds I. Nachamkin and M. J. Blaser (Washington, DC: ASM Press), 155-175.

Neogi, P. K., and Shahid, N. S. (1987) Serotypes of Campylobacter jejuni isolated from patients attending a diarrhoeal disease hospital in urban Bangladesh. J. Med. Microbiol. 24, 303-307.

Nielsen, E. M., Engberg, J., and Madsen, M. (1997). Distribution of serotypes of Campylobacter jejuni and C. coli from Danish patients, poultry, cattle and swine. FEMS Immunol. Med. Microbiol. 19, 47-56.

Nielsen, E. M., Fussing, V., Engberg, J., Nielsen, N. L., and Neimann, J. (2006). Most Campylobacter subtypes from sporadic infections can be found in retail poultry products and food animals. Epidemiol. Infect. 134, 758-767.

Oria, R. B., Patrick, P. D., Oria, M. O., Lorntz, B., Thompson, M. R., Azevedo, O. G., Lobo, R. N., Pinkerton, R. F., Guerrant, R. L., and Lima, A. A. (2010). ApoE polymorphisms and diarrheal outcomes in Brazilian shanty town children. Braz. J. Med Biol. Res. 43, 249-256.

Owen, R. J., Fitzgerald, C., Sutherland, K., and Borman, P. (1994). Flagellin gene polymorphism analysis of Campylobacter jejuni infecting man and other hosts and comparison with biotyping and somatic antigen serotyping. Epidemiol. Infect. 113, 221-234

Oza, A. N., Thwaites, R. T., Wareing, D. R., Bolton, F. J., and Frost, J. A. (2002). Detection of heat-stable antigens of Campylobacter jejuni and C. coli by direct agglutination and passive hemagglutination. J. Clin. Microbiol. 40, 996-1000.

Palyada, K., Threadgill, D., and Stintzi, A. (2004). Iron acquisition and regulation in Campylobacter jejuni. J. Bacteriol. 186, 4714-4729.

Parkhill, J., Wren, B. W., Mungall, K. Ketley, J. M., Churcher, C., Basham, D., Chillingworth, T., Davies, R. M., Feltwell, T., Holroyd, S., Jagels, K., Karlyshev, A. V., Moule, S. Pallen, M. J., Penn, C. W., Quail, M. A., Rajandream, M. A., Rutherford, K. M., van Vliet, A. H. M. Whitehead, S., and Barrell, B. G. (2000). The genome sequence of the food-borne pathogen Campylobacter jejuni reveals hypervariable tracts. Nature 403, 665-668.

Patton, C. M., Barrett, T. J., and Morris, G. K. (1985). Comparison of the Penner and Lior methods for serotyping Campylobacter spp. J. Clin. Microbiol. 22, 558-565.

Pearson, B. M., Pin, C., Wright, J. I'Anson, K., Humphrey, T., and Wells, J. M. (2004). Comparative genome analysis of Campylobacter jejuni using whole genome DNA microarrays. FEBS Lett. 554 224-230.

Penner, J. L., and Hennessy, J. N. (1980). Passive hemagglutination technique for serotyping Campylobacter fetus subsp. jejuni on the basis of soluble heat-stable antigens. J. Clin. Microbiol. 12, 732-737.

Penner, J. L., Hennessy, J. N., and Congi, R. V. (1983). Serotyping of Campylobacter jejuni and Campylobacter coli on the basis of thermostable antigens. Eur. J. Clin. Microbiol. 2, 378-383.

Petersen, L., Larsen, T. S., Ussery, D. W. On, S. L. W., and Krogh, A. (2003). RpoD promoters in Campylobacter jejuni exhibit a strong periodic signal instead of a -35 box. J. Mol. Biol. 326, 1361-1372.

Poly, F., Serichatalergs, O., Schulman, M., Ju, J., Cates, C. N., Kanipes, M. I., Mason, C., and Guerry, P. (2011). Discrimination of major capsular types of Campylobacter jejuni by multiplex PCR. J. Clin. Microbiol.49, 1750-1757.

Pope, J. E., Krizova, A., Garg, A. X., Thiessen-Philbrook, H., and Ouimet, J. M. (2007). Campylobacter reactive arthritis: a systemic review. Semin. Arthritis Rheum. 37, 48-55.

Preston, M. A., and Penner, J. L. (1989). Characterization of cross-reacting serotypes of Campylobacter jejuni. Can. J. Microbiol. 35, 265-273.

Ravel, A., Nesbitt, A., Marshall, B., Sittler, N., Pollari, F. (2011). Description and burden of travel-related cases by enteropathogens reported in a Canadian community. J. Travel Med. 18, 8-19.

Reid, A. N., Pandey, R., Palyada, K., Naikare, H., and Stinzi, A. (2008). Identification of Campylobacter jejuni genes involved in the response to acidic $\mathrm{pH}$ and stomach transit. Appl. Environ. Microbiol. 74 1583-1597.

Riddle, M. S., Sanders, J. W., Putnam, S. D., and Tribble, D. R. (2006). Incidence, etiology, and impact of diarrhea among long-term travelers (US military and similar populations): a systemic review. Am. J. Trop. Med. Hyg. 74, 891-900.

Rijkers, G. T., Sanders, E. A., Breukels, M. A., and Zegers, B. J. (1998) Infant B cell responses to polysaccharide determinants. Vaccine 16, 1396-1400.

Rodriguez, L. A., and Ruigomez, A. (1999). Increased risk of inflammatory bowel syndrome after bacterial gastroenteritis: cohort study. $\mathrm{Br}$. Med. J. 318, 565-566.

Rodriguez, L. A., Ruigomez, A., and Panes, J. (2006). Acute gastroenteritis is followed by an increased risk of inflammatory bowel disease. Gastroenterology 130, 1588-1594.

Rose, A., Kay, E., Wren, B. W., and Dallman, M. J. (2011). The Campylobacter jejuni NCTC 11168 capsule prevents excessive cytokine production by dendritic cells. Med. Microbiol. Immunol. doi: 10.1007/500430-0110214-1

Santos, D. N., Assis, A. M., Bastos, A. C., Santos, L. M., Santos, C. A., Strina, A., Prado, M. S., AlmeidaFiho, N. M., Rogrigues, L. C., and Barreto, M. L. (2008). Determinants of cognitive function in childhood: a cohort study in a middle income context. BMC Public Health 8, 202. doi:10.1186/1471-2458-8-202

Scallan, E. H. R., Angulo, F. J., Tauxe, R. V., Widdowson, M. A., Roy, S. L. Jones, J. L., and Griffin, P. M. (2011). Foodborne illness acquired in the 
United States - major pathogens. Emerging Infect. Dis. 17, 7-15.

Shah, N., DuPont, H. L., and Ramsey, D. J. (2009). Global etiology of travelers' diarrhea: systematic review from 1973 to the present. Am. J. Trop. Med. Hyg. 80, 609-614.

Siemer, B. L., Harrington, C. S., Nielsen, E. M., Borck, B., Nielsen, N. L., Engberg, J., and On, S. L. (2004). Genetic relatedness among Campylobacter jejuni serotyped isolates of diverse origin as determined by numerical analysis of amplified fragment length polymorphism (AFLP) profiles. J. Appl. Microbiol. 96, 795-802.

Sjogren, E., Johny, M., and Kaijser, B. (1989). The serotype distribution of Campylobacter in patients with diarrhoea in Kuwait. FEMS Microbiol. Lett. 48, 237-239.

Skirrow, M. B., Jones, D. M., Sutcliffe, E., and Benjamin, J. (1993). Campylobacter bacteraemia in England and Wales, 1981-91. Epidemiol. Infect. 110, 567-573.

Soofi, S. B., Habib, M. A., von Seidlein, L., Khan, M. J., Muhammad, S., Bhutto, N., Khan, M. I., Rasool, S., Zafar, A., Clemens, J. D., Nizami, Q., and Bhutta, Z.A. (2011). A comparison of disease caused by Shigella and
Campylobacter species: 24 months community based surveillance in 4 slums of Karachi, Pakistan. J. Infect. Public Health 4, 12-21.

Sorvillo, F. J., Lieb, L. E., and Waterman, S. H. (1991). Incidence of campylobacteriosis among patients with AIDS in Los Angeles County. J. Acquir. Immun. Defic. Syndr. 4, 598.

Stintzi, A. (2003). Gene expression profile of Campylobacter jejuni in response to growth temperature variation. J. Bacteriol. 185, 2009-2016.

Tam, C. C., Rodrigues, L. C., Viviani, L., Dodds, J. P., Evans, M. R., Hunter, P. R., Gray, J. J., Letley, L. H., Rait, G., Tompkins, D. S., O’Brien, S. J., and On behalf of the IID2 Study Executive Committee. (2012). Longitudinal study of infectious intestinal disease in the UK (IID2 study): incidence in the community and presenting to general practice. Gut. 61, 69-77.

Tarleton, J. L., Haque, R., Mondal, D., Shu, J., Farr, B. M., and Petri, W. A Jr. (2006). Cognitive effects of diarrhea, malnutrition, and Entamoeba histolytica infection on school age children in Dhaka, Bangladesh. Am. J. Trop. Med. Hyg. 74, 475-481.
Tee, W., and Mijch, A. (1998). Campylobacter jejuni bacteremia in human immunodeficiency virus (HIV)infected and non-infected patients: comparison of clinical features and review. Clin. Infect. Dis. 26, 91.

Thornley, J. P., Jenkins, D., and Neal, K. (2001). Relationship of Campylobacter toxigenicity in vitro to the development of post-infectious inflammatory bowel syndrome. J. Infect. Dis. 184, 606.

Vimr, E. R., and Steenbergen, S. M. (2009). Early molecular-recognition events in the synthesis and export of group 2 capsular polysaccharides. Microbiology 155, 9-15.

Wareing, D. R., Bolton, F. J., Fox, A. J., Wright, P. A., and Greenway, D. L. (2002). Phenotypic diversity of Campylobacter isolates from sporadic cases of human enteritis in the UK. J. Appl. Microbiol. 92, 502-509.

Woodward, D. L., and Rodgers, F. G. (2002). Identification of Campylobacter heat-stable and heat-labile antigens by combining the Penner and Lior serotyping schemes. J. Clin. Microbiol. 40, 741-745.

Zilbauer, M., Dorrell, N., Boughan, P. K., Harris, A., Wren, B. W., Klein, N. J., and Bajaj-Elliott, M. (2005). Intestinal innate immunity to Campylobacter jejuni results in induction of bacteriocidal human beta-defensins 2 and 3. Infect. Immun. 73, 7281-7289.

Conflict of Interest Statement: The authors declare that the research was conducted in the absence of any commercial or financial relationships that could be construed as a potential conflict of interest.

Received: 29 November 2011; accepted: 24 January 2012; published online: 15 February 2012

Citation: Guerry P, Poly F, Riddle M, Maue AC, Chen Y-H and Monteiro MA (2012) Campylobacter polysaccharide capsules: virulence and vaccines. Front. Cell. Inf. Microbio. 2:7. doi: 10.3389/fcimb.2012.00007

Copyright () 2012 Guerry, Poly, Riddle, Maue, Chen and Monteiro. This is an open-access article distributed under the terms of the Creative Commons Attribution Non Commercial License, which permits non-commercial use, distribution, and reproduction in other forums, provided the original authors and source are credited. 